\title{
Detección de síncope vasovagal con adaptación de la Escala de Calgary en estudiantes universitarios colombianos
}

\author{
Using a Calgary Scale adaptation to detect vasovagal syncope in Colombian university students \\ Karen Dayanna Penagos-Guio' orcid.org/0000-0002-3065-3016 \\ Alejandra Sandoval-Montealegre ${ }^{1}$ orcid.org/0000-0002-7452-1410 \\ Diana Goretty Llano-Bautista' ${ }^{1}$ orcid.org/0000-0003-4471-7268 \\ José Julián Galindo-Martínez' orcid.org/0000-0001-8227-2103 \\ Leonardo José Reyes-Ortiz'1 orcid.org/0000-0003-0443-1767 \\ Yaliana Tafurt-Cardona ${ }^{*}$ orcid.org/0000-0003-1289-2395
}

1. Fundación Universitaria Navarra-UNINAVARRA. Facultad de Ciencias de la Salud. Neiva, Colombia

Penagos-Guio KD, Sandoval-Montealegre A, Llano-Bautista G, Galindo-Martínez JJ, Reyes-Ortiz LJ, Tafurt-Cardona Y. Detección de síncope vasovagal con una adaptación de la Escala de Calgary en estudiantes de una universidad del sur de Colombia. Univ. Salud. 2021;23(2):92-99. DOI: https://doi.org/10.22267/rus.212302.220

\section{Resumen}

Introducción: El síncope vasovagal tiene una incidencia entre 10\% y 19\% en la población general, el primer episodio suele aparecer en la juventud. Objetivo: Estimar la prevalencia de síncope vasovagal en una población universitaria del sur de Colombia, utilizando una adaptación de la Escala de Calgary como cuestionario diagnóstico. Materiales y métodos: Después de la firma voluntaria del consentimiento informado, los estudiantes fueron entrevistados y se les aplicó la Escala de Calgary. Posteriormente, se realizó un cuestionario de seguimiento a los participantes positivos. Resultados: Participaron 280 estudiantes, el $17 \%$ obtuvo un puntaje positivo para síncope vasovagal. La edad media fue $21,45 \pm 5,18$; con mayor positividad para el género femenino. En el seguimiento, el $10 \%$ de los positivos, consultó a urgencias por pérdida de conciencia, $4 \%$ fue ingresado a hospitalización y 10\% presentó lesiones secundarias. Conclusiones: Un número representativo de participantes fue positivo en la Escala de Calgary, ninguno de ellos había recibido diagnóstico de síncope neuralmente mediado, pese a haber consultado a urgencias y/o presentar lesiones secundarias por pérdida de conciencia. Incluir la Escala de Calgary en la evaluación inicial del paciente consultante por pérdida de conciencia, podría ayudar al diagnóstico temprano de síncope vasovagal.

Palabras clave: Síncope; síncope vasovagal; encuestas y cuestionarios; encuestas epidemiológicas. (Fuente: DeCS, Bireme).

\begin{abstract}
Introduction: Vasovagal syncope has an incidence between 10\% and 19\% in the general population, whose first episodes appear in youth. Objective: To estimate the prevalence of vasovagal syncope in a university student population from the southern Colombia, using a diagnostic questionnaire adapted from the Calgary Scale. Materials and methods: After voluntary signing of the informed consent form, students were surveyed through the Calgary Scale, and a subsequent follow-up questionnaire was conducted on positive participants. Results: 280 students participated in the study and $17 \%$ of them obtained a positive score for vasovagal syncope, being positive female participants the largest group. The mean age of participants was $21.45 \pm 5.18$ years. At the follow-up, $10 \%$ of the positive cases had sought out treatment in emergency rooms because of consciousness loss, $4 \%$ were hospitalized, and 10\% had secondary injuries. Conclusions: Even though an important number of patients was positive on the Calgary Scale, none of them had been diagnosed with neutrally mediated syncope, despite being treated in emergency rooms and/or presenting secondary lesions due to consciousness loss. The use of the Calgary Scale during the assessment of patients being treated for consciousness loss could help to early diagnose vasovagal syncope.
\end{abstract}

Keywords: Syncope/diagnosis; vasovagal syncope; surveys and questionnaires; epidemiologic methods. (Source: DeCS, Bireme).

*Autor de correspondencia

Yaliana Tafurt-Cardona

e-mail: yalianat@gmail.com 


\section{Introducción}

Se denomina síncope a la pérdida temporal de conciencia $y$ tono postural causado por hipoperfusión cerebral global transitoria (HCGT),

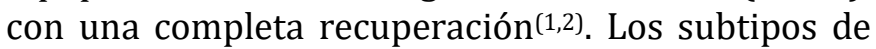
síncope más comunes son el síncope neuralmente mediado y la hipotensión ortostática ${ }^{(1,2)}$. Se ha reportado que la probabilidad de presentar un episodio sincopal durante la vida es del $50 \%(1,3-5)$; estudios realizados por el grupo de Calgary $y$ Amsterdam afirman que un gran porcentaje de individuos mayores de 60 años han presentado uno o más episodios de síncope en su vida, con mayor frecuencia en mujeres (42\%) que en varones $(31 \%)^{(6)}$. En Colombia, se ha descrito una prevalencia global entre el $40 \%$ al $50 \%$ en pacientes jóvenes, en mayores de 65 años las cifras son cercanas al $28 \%$, especialmente en el sexo femenino $(62,7 \%)^{(7)}$.

Actualmente, se utiliza el Test de mesa basculante como prueba diagnóstica para síncope vasovagal, con una sensibilidad entre el $20 \%$ al $74 \%(8)$. Esta prueba tiene una especificidad cercana al 90\%, porcentaje que disminuye con el uso de ciertos fármacos y la de ser necesario la utilización de un mayor ángulo de inclinación en la mesa. La mesa basculante tiene criterios específicos para su aplicación como son: episodio único que no se relaciona a síncope con alto riesgo para el paciente; episodios recurrentes sin cardiopatía estructural; para distinguir síncope convulsivo de epilepsia; cuando la evaluación inicial no es diagnóstica y se sospecha hipotensión ortostática, para el diagnóstico de pseudo síncope ${ }^{(1,2,6,9)}$ y una vez excluido el síncope cardiogénico en pacientes con cardiopatía estructural $(1,2)$.

La mesa basculante es positiva cuando causa pérdida transitoria de la conciencia asociada a hipotensión con o sin bradicardia/asistolia $(2,6)$, si bien su positividad indica una alteración del reflejo vagal, un resultado negativo no excluye su diagnóstico $(1,2,6,9)$. Piccirillo et al., encontraron pacientes que presentaban alteraciones en el electrocardiograma registrado durante la prueba de la mesa basculante y que incluían un intervalo P-PR reducido, desviaciones estándar más altas del intervalo R-R y asistolia previo al síncope ${ }^{(10)}$, hallazgos que pueden sugerir la coexistencia de cardiopatía y síncope vasovagal.
Existen alternativas a la mesa basculante para la identificación de síncope vasovagal, entre estas la Escala de Calgary(8,11,12), con sensibilidad del $89 \%$ y especificidad del 91\%, aprobada por la Sociedad Europea de Cardiología como cuestionario diagnóstico, desarrollado para pacientes sin anomalías cardiacas estructurales(8,11,12). La escala consiste en siete preguntas específicas de la historia médica, desencadenantes, signos o síntomas presentados por el afectado durante un posible cuadro sincopal, cada ítem afirmativo confiere un puntaje entre $(-5)$ y $(+3)$, según qué tanto oriente o no hacia una etiología neuralmente mediada. Al finalizar la encuesta se suman o restan los puntajes obtenidos calculando una puntuación total que va desde $(-14)$ a $(+6)^{(8,11,12)}$. El resultado se interpreta así: obtener un puntaje en la escala $\geq(-2)$ sugiere un origen vasovagal del episodio sincopal y un valor $(<2)$ indica que el síncope tiene otra causa $a^{(8,11,12)}$.

La Escala de Calgary ${ }^{(8,11,12)}$ no se encuentra incluida dentro de las guías internacionales para diagnóstico y manejo de síncope ${ }^{(1,2)}$, pero ha sido utilizada para confirmar o excluir síncope vasovagal en la evaluación de nuevas pruebas diagnósticas para síncope ${ }^{(13)}$. A diferencia de la mesa basculante, aplicar la escala no representa intervenciones en el paciente y puede incluirse dentro de la anamnesis, permitiendo identificar o descartar rápidamente el cuadro vasovagal, así implementar un manejo eficiente hacia un pronóstico favorable que prevenga complicaciones a corto y largo plazo secundarias a las pérdidas de conciencia(12,14,15); lo anterior sumado a la representativa sensibilidad y especificidad de esta escala ${ }^{(8)}$, la convierten en una herramienta costo efectiva cuya implementación agilizaría el abordaje médico, beneficiando al paciente con síncope ${ }^{(3)}$.

Los episodios sincopales de aparición repentina o frecuente pueden causar deterioro en la calidad de vida, dado su alto riesgo de trauma, especialmente en pacientes de edad avanzada o con antecedentes de patología cardiovascular(7). Estudios previos han indicado que el síncope genera entre el $0,8 \%$ y el $2,4 \%$ de ingresos al servicio de urgencias $(1,2,6,16)$, su etiología sólo es determinable durante la evaluación inicial en $40 \%$ a $70 \%$ de los $\operatorname{casos}^{(1,17)}$; al ser el síncope una afección común, la identificación temprana de su etiología y un manejo adecuado para el mismo optimizaría el manejo en los centros de atención de salud beneficiando directamente a los pacientes. Por tal motivo, este estudio tuvo como 
objetivo estimar la prevalencia de síncope vasovagal en una población universitaria del sur de Colombia, utilizando una adaptación de la Escala de Calgary ${ }^{(8,11,12)}$ como cuestionario diagnóstico.

\section{Materiales y métodos}

Estudio descriptivo de corte transversal, se realizó en estudiantes mayores de 18 años de edad, de la Facultad de Ciencias de la Salud de la Fundación Universitaria Navarra-UNINAVARRA, pertenecientes a los programas de medicina, enfermería y tecnología en radiología e imágenes diagnósticas, durante el primer período del año 2018. La población objeto de estudio fue definida como estudiantes de dicha institución de los diferentes programas. Los criterios de exclusión fueron estudiantes con diagnóstico conocido de síncope, enfermedad cardiovascular asociada y/o consumo de medicamentos antihipertensivos. Después de la firma voluntaria del consentimiento informado (según lo dispuesto en la Resolución No. 8430 de 1993 del Ministerio de Salud), se les aplicó a los participantes la Escala de Calgary $(8,11,12)$.

Un año después de la aplicación de la escala, se contactó nuevamente a los participantes que fueron positivos para síncope vasovagal según esta evaluación (48 en total), con el fin de informarles sobre su resultado, también se les realizó una encuesta de seguimiento específico interrogando sobre episodios sincopales, características y tipo de atención recibida en caso de haber consultado a un centro de salud por dicha causa.

\section{Análisis estadístico}

El análisis de datos se realizó con el paquete estadístico $R$-commander. Las variables continuas se expresaron con la media \pm desviación estándar y las variables cualitativas en frecuencias y proporciones. Se realizó una prueba de chi-cuadrado $\left(X^{2}\right)$ para la comparación de proporciones. Se utilizó la calculadora estadística Online Openepi gratuita para calcular la muestra representativa del total de estudiantes de una Facultad de Ciencias de la Salud que incluía: Estudiantes de medicina (473), estudiantes de enfermería (429) y estudiantes de tecnología en radiología e imágenes diagnósticas (116), indicando un tamaño de muestra aleatorizado de 280 estudiantes (software Epidat 4.1 licencia libre), con un intervalo de confianza del 95\%.

\section{Consideraciones éticas}

La participación de los estudiantes fue voluntaria previa explicación de procedimientos y firma de consentimiento informado. Todos los procedimientos y protocolos utilizados en el estudio fueron aprobados por el Comité de Ética de la Fundación Universitaria Navarra-UNINAVARRA, adoptando los principios bioéticos establecidos en la Declaración de Helsinki de la Asociación Médica Mundial (AMM) principios éticos para las investigaciones médicas en seres humanos(18). Los cuestionarios fueron codificados para proteger la confidencialidad de las participantes.

\section{Resultados}

Previo a la aplicación de las encuestas, se realizó una prueba piloto para evaluar la practicidad del cuestionario. Un total de 280 estudiantes participaron del estudio, 7 encuestas fueron excluidas por repetición. La Tabla 1 ilustra las características sociodemográficas de la población, indicando que $17 \%$ de los participantes obtuvo un puntaje positivo para síncope vasovagal, quienes presentaron una edad media de 21,45 $\pm 5,18$, con mayor frecuencia en el género femenino (12\%). El $13 \%$ de los participantes positivos presentaron un Índice de Masa Corporal normal (IMC: 18,5-24,9), con antecedentes de bloqueo fascicular 0 bifascicular, ausencia de actividad eléctrica (asistolia), taquicardia supraventriular o Diabetes Mellitus según la escala.

La Tabla 2 indica el porcentaje de respuestas afirmativas según la Escala de Calgary(15,16). Sin embargo, no hubo diferencias significativas entre los grupos.

El seguimiento realizado en la población positiva incluyó 30 participantes (65\% del total), $12 \%$ no pudo ser contactado, y 15\% se negó a participar. La Tabla 3 indica los datos, del cuestionario de seguimiento para estudiantes positivos según la Escala Calgary, indicando que el $10 \%$ consultaron a urgencias por episodios de pérdida de conciencia, el $4 \%$ fue ingresado a hospitalización por la misma causa, y el 10\% habían presentado algún tipo de lesión secundaria a pérdida de la conciencia; al 7\% de los participantes le fue practicado un test de mesa basculante u otros exámenes diagnósticos, y sólo el $3 \%$ obtuvo un resultado positivo en la prueba. 
La Figura 1 indica el manejo que recibieron los participantes positivos al consultar a urgencias $10 \%$ consultó a urgencias 5\% requirieron hospitalización ninguno recibió información sobre medidas preventivas o fármacos, al $5 \%$ le habían realizado el test de Mesa Basculante u otros exámenes y $5 \%$ obtuvo un resultado positivo en la Mesa Basculante.

Tabla 1. Características sociodemográficas de la población estudio

\begin{tabular}{|c|c|c|c|}
\hline Variable & $\begin{array}{c}\text { Positivos } \\
\text { n (\%) }\end{array}$ & $\begin{array}{l}\text { Negativos } \\
\text { n (\%) }\end{array}$ & $p$ \\
\hline Total población & $46(17)$ & $227(83)$ & \\
\hline \multicolumn{4}{|l|}{ Edad } \\
\hline Media \pm DS & $21,45 \pm 5,18$ & $20,94 \pm 3,59$ & $0,072^{*}$ \\
\hline \multicolumn{4}{|l|}{ Género } \\
\hline Femenino & $32(12)$ & $142(52)$ & \\
\hline Masculino & $14(5)$ & $85(31)$ & $0,080^{+}$ \\
\hline \multicolumn{4}{|l|}{ Estado civil } \\
\hline Soltero & $42(15)$ & $208(76)$ & \\
\hline Casado & $1(0)$ & $4(2)$ & \\
\hline Unión libre & $2(1)$ & $13(5)$ & \\
\hline Divorciado & $1(0)$ & $2(1)$ & \\
\hline \multicolumn{4}{|l|}{ Ocupación } \\
\hline Estudia solamente & $41(15)$ & $201(74)$ & \\
\hline Estudia y trabaja & $5(2)$ & $26(9)$ & $0,701^{\dagger}$ \\
\hline \multicolumn{4}{|l|}{ IMC } \\
\hline Delgadez extrema (IMC:16-16.9) & $0(0)$ & $3(1)$ & \\
\hline Delgadez (IMC:17-18.4) & $0(0)$ & $8(3)$ & \\
\hline Normal (IMC:18.5-24.9) & $33(12)$ & $146(53)$ & \\
\hline Sobrepeso (IMC:25-29.9) & $11(4)$ & $58(21)$ & \\
\hline Obesidad Grado I(IMC:30-34.9) & $2(1)$ & $10(4)$ & \\
\hline Obesidad Grado II(35-39.9) & $0(0)$ & $2(1)$ & $0,701^{\dagger}$ \\
\hline \multicolumn{4}{|l|}{ Antecedentes $s^{\ddagger}$} \\
\hline $\mathrm{Si}$ & $6(13)$ & 40 (87) & \\
\hline No & $40(18)$ & $187(82)$ & $0,449 \dagger$ \\
\hline
\end{tabular}

DS: Desviación estándar; P: probabilidad; *: Prueba de ANOVA para la comparación de medias; †: Prueba de Chi-cuadrado para la comparación de proporciones, ‡: Bloqueo fascicular o bifascicular, asistolia, Taquicardia supraventriular o Diabetes Mellitus.

Tabla 2. Porcentaje de positividad según la Escala Calgary

\begin{tabular}{|c|c|c|c|c|}
\hline & $\begin{array}{c}\text { Puntaje } \\
n\end{array}$ & $\begin{array}{c}\text { Positivos } \\
\text { n (\%) }\end{array}$ & $\begin{array}{c}\text { Negativos } \\
\text { n (\%) }\end{array}$ & $p$ \\
\hline Total población & & $46(17)$ & $227(83)$ & $0,100^{*}$ \\
\hline \multicolumn{5}{|c|}{ Mareos desmayos cuando está de pie prolongadamente } \\
\hline $\mathrm{Si}$ & 1 & $28(10)$ & $9(3)$ & \\
\hline No & 0 & $18(7)$ & $218(80)$ & $0,512^{*}$ \\
\hline \multicolumn{5}{|c|}{ Sudoración o calor previo al Síncope } \\
\hline $\mathrm{Si}$ & 2 & $23(8)$ & $6(3)$ & \\
\hline No & 0 & $23(8)$ & $221(81)$ & $0,130^{*}$ \\
\hline \multicolumn{5}{|c|}{ Mareos o desmayos con dolor/ maniobras médicas } \\
\hline $\mathrm{Si}$ & 3 & $9(3)$ & $1(0)$ & \\
\hline No & 0 & $37(14)$ & $226(83)$ & $0,786^{*}$ \\
\hline \multicolumn{5}{|c|}{ Recuerda algo mientras estaba inconsciente } \\
\hline $\mathrm{Si}$ & -2 & $11(4)$ & $7(3)$ & \\
\hline No & 0 & $35(13)$ & $220(80)$ & $0,501^{*}$ \\
\hline \multicolumn{5}{|c|}{ Si hubo testigos notaron cianosis durante el desmayo } \\
\hline $\mathrm{Si}$ & -4 & $3(1)$ & $3(1)$ & \\
\hline No & 0 & $43(16)$ & $224(82)$ & $0,265^{a}$ \\
\hline \multicolumn{5}{|c|}{ Los episodios de síncope empezaron luego de los 35 años } \\
\hline $\mathrm{Si}$ & -3 & $0(0)$ & $0(0)$ & \\
\hline No & 0 & $46(17)$ & $227(83)$ & \\
\hline
\end{tabular}

Porcentaje de positividad para los ítems interrogados de la Escala de Calgary(8,11-12,19); * Prueba de Chi-cuadrado para la comparación de proporciones 
Tabla 3. Cuestionario de seguimiento para estudiantes positivos según la Escala Calgary

\begin{tabular}{lc}
\hline \multicolumn{1}{c}{ Variable } & $\begin{array}{c}\text { Cantidad } \\
\text { n (\%) }\end{array}$ \\
\hline Total participantes & $30(100)$ \\
Género & $9(30)$ \\
Masculino & $21(70)$ \\
Femenino & \\
Edad Media \pm DS & $30(100)$ \\
21 años & $29(96)$ \\
Estado civil & $1(4)$ \\
Soltero & $26(86)$ \\
Casado & $4(14)$ \\
Ocupación & \\
Estudia solamente & $17(56)$ \\
Estudia y trabaja & $13(44)$ \\
Escala de Calgary & \\
Positivo & $8(27)$ \\
Negativo & $22(73)$ \\
Ha tenido episodios de pérdida de la conciencia & $0(0)$ \\
Si & $0(0)$ \\
No & Cuándo fue la última vez que tuvo un episodio de pérdida de la conciencia? \\
3 días previos & $0(0)$ \\
Esta semana & $0(0)$ \\
Último mes & $7(23)$ \\
Tres meses previos & $1(4)$ \\
Antes meses previos & $22(73)$ \\
No aplica & \\
¿Ha tenido lesiones, fracturas o trauma secundarios a episodios de pérdida de la conciencia? & $3(10)$ \\
Si & $27(90)$ \\
\hline
\end{tabular}

DS: Desviación estándar

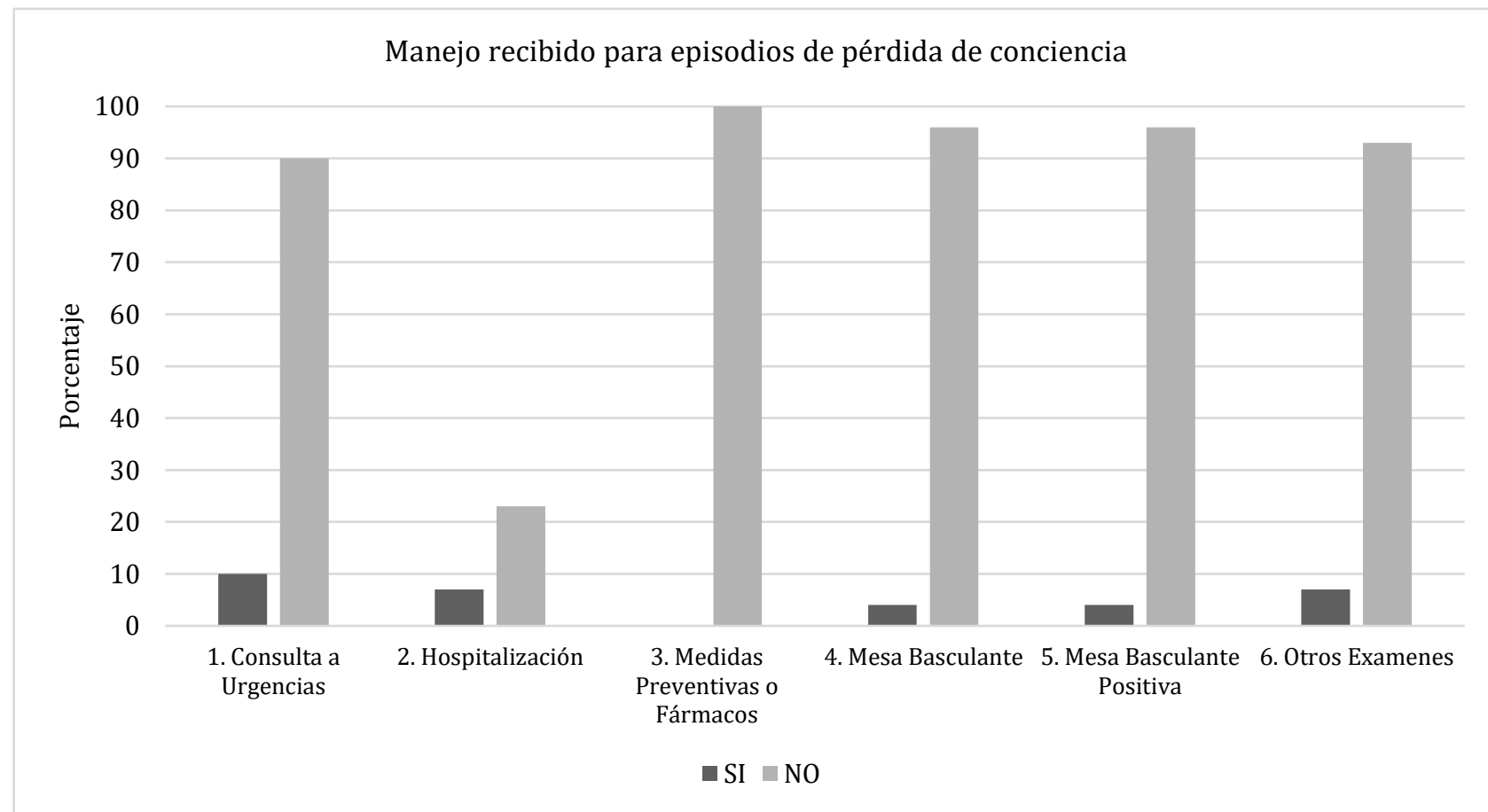

Figura 1. Porcentaje de participantes a quienes se les realizó cuestionario de seguimiento que: 1 . Consultaron a urgencias por un episodio de pérdida de conciencia; 2. Requirieron hospitalización por episodio de pérdida de la conciencia; 3. Recibieron información sobre medidas preventivas o fármacos para manejo de síncope vasovagal; 4. Les fue realizada una prueba de Mesa Basculante; 5. Obtuvieron un resultado positivo en la prueba de Mesa Basculante; 6 Les fue realizado otros estudios diagnósticos. 


\section{Discusión}

Incluir la escala de Calgary(11,13,16,19) como una herramienta adicional dentro de la anamnesis puede facilitar y agilizar el diagnóstico de síncope desde la primera atención del paciente, puesto que, la prevalencia estimada de síncope vasovagal es de aproximadamente un $13 \%$ a $40 \%$ con mayor frecuencia en la población joven(2,3,5,6,20-23).

La edad promedio para los positivos fue entre $21 \mathrm{y}$ 26 años, especialmente en el género femenino. Diferentes estudios, concuerdan que el síncope se puede manifestar por primera vez en distintas edades, el primer episodio puede aparecer tanto a los 20,60 como a los 80 años de edad(1), con una tasa de recurrencia del $30 \%$ cada 2 años para pacientes con diagnóstico establecido(7). Baker et al.,(14) sugieren que mujeres jóvenes tienen mayor vasodilatación mediada por receptores adrenérgicos $\beta$ que suele compensar y/o mitigar la vasoconstricción adrenérgica $\alpha$, esto explicaría los frecuentes fenómenos de ortostatismo(8).

El uso de anticonceptivos orales (ACO) aumenta la vasodilatación mediada por el adrenoreceptor $\beta$ por cuanto se relacionan con cifras tensionales elevadas especialmente si contienen concentraciones altas de estradiol(11); cantidades elevadas de progesterona se asocian con menor respuesta vasoconstrictora ante un estímulo simpático(6,12,17), sin embargo, el uso de anticonceptivos hormonales no fue interrogado en este estudio(12,14,21,22).

La escala de Calgary, evalúa la existencia de antecedentes cardiovasculares como bloqueo fascicular o bifascicular, asistolia, entre otros, que busca excluir aquellos pacientes con alta probabilidad de síncope cardiogénico. Estas patologías resultan confusas para muchos participantes, dado que no las identifican con certeza, por lo tanto, puede ser una pregunta fuente de sesgo por confusión. En el presente estudio el $13 \%$ de los participantes positivos para la escala refirió alguno de los antecedentes previamente descritos, datos que concuerdan con los hallazgos de alteraciones cardiovasculares eléctricas en pacientes con síncope vasovagal descritos por Piccirilo et al.,(10).

Algunas situaciones suelen anteceder al síncope vasovagal como: posición continua de bipedestación $>30$ segundos $^{(9,20)}$, estrés o emociones fuertes, exposición al dolor, esfuerzo, maniobras médicas o sangrado(9,21). Estar sentado o de pie por tiempo prolongado fue la situación más reiterada por los participantes positivos para síncope. Otros síntomas descritos, previos al síncope (pródromos): sudoración, palidez, náuseas, aturdimiento, calor, visión en túnel y molestias abdominales han sido descritos en la literatura(1,2,6,23,24). En este estudio la sudoración y/o calor fue de los síntomas más frecuentes en la población objeto de estudio.

El síncope es un motivo frecuente de consulta a nivel mundial $(8,9,11)$, lo cual concuerda con los hallazgos en participantes positivos a quienes se les hizo seguimiento; dado que el $10 \%$ acudió al servicio de urgencias, de los cuales el $4 \%$ culminó en hospitalización, incluso el $10 \%$ sufrió lesiones secundarias a la pérdida de conciencia.

También se evidenció que el test de mesa basculante continúa siendo utilizado como prueba diagnóstica de elección, aun cuando las guías internacionales de síncope no sugieren su uso, resaltando que una prueba negativa no excluye síncope vasovagal(1,2,6,9,25). Lo anterior evidencia en la actualidad el poco o errado abordaje proporcionado al síncope vasovagal, sin tener en cuenta las consecuencias que pueden generar los episodios sincopales repetitivos, que van más allá de la lesión física, tales como depresión y ansiedad por no tener un apropiado diagnóstico y manejo(26).

Según las directrices de la Heart Rhythm Society 2015 cerca de $60 \%$ de los casos de síncope vasovagal pueden ser diagnosticados con la historia clínica, examen físico y electrocardiograma inicial para descartar el síncope cardiogénico, el cual representa un riesgo potencial para la vida del paciente $(6,8,27,28)$. $\mathrm{Si}$ el cuadro clínico sugiere síncope vasovagal, la mesa basculante no aporta información adicional y el uso exclusivo de la Escala de Calgary $(8,9,11)$ dentro de la anamnesis, proveerá un diagnóstico orientador en casi todos los $\operatorname{casos}^{(1-3,6,13)}$.

\section{Conclusiones}

El presente estudio da a conocer la prevalencia de síncope vasovagal en una población universitaria del sur de Colombia, utilizando una adaptación de la Escala de Calgary como cuestionario diagnóstico. La frecuencia de estudiantes con síncope fue de 17\%, lo cual sugiere que esta condición clínica puede evolucionar progresivamente en la población joven. 
La adaptación de la Escala de Calgary utilizada, indicó que es una herramienta útil de diagnóstico, no invasiva y efectiva, con orientación preventiva frente a esta patología. El seguimiento realizado evidencia que la mayoría de los individuos estudiados positivos para síncope vasovagal no han recibido un diagnóstico, a pesar de haber acudido a la consulta de urgencias, con ingresos hospitalarios e incluso presentar lesiones secundarias a los episodios de pérdida de conciencia.

Además, demuestra la necesidad para la población en riesgo y sus familiares, de conocer e identificar los factores desencadenantes del episodio sincopal y síntomas como sudoración y/o pródromos que anticipan al síncope vasovagal, así disponer del tiempo necesario para evitar situaciones de riesgo como escaleras, ventanas, entre otros; que podrían culminar en lesiones graves al tener pérdida de la conciencia.

Por lo anterior, se sugiere frente al reporte positivo para síncope vasovagal, realizar seguimiento con orientación preventiva en esta población, considerando la posibilidad de caídas que conlleven a trauma con deterioro en la calidad de vida de los afectados. También, es pertinente realizar estudios que incluyan un mayor número de pacientes, con una descripción más específica de los episodios sincopales presenciados por el personal de salud. Estos resultados brindan información valiosa a las instituciones de salud al momento de realizar un diagnóstico oportuno, y un tratamiento eficaz.

\section{Referencias}

1. Shen WK, Sheldon RS, Benditt DG, Cohen MI, Forman DE, Goldberger ZD, et al. 2017 ACC/AHA/HRS guideline for the evaluation and management of patients with syncope: Executive summary: A report of the american college of cardiology/american heart association task force on clinical practice guidelines, and the heart rhythm society. Circulation. 2017;136(5):e60-e122. Disponible en: https://doi.org/10.1161/cir.0000000000000499

2. Brignole M, Moya Á, de Lange FJ, Deharo JC, Elliott PM, Fanciulli A, et al. Guía ESC 2018 sobre el diagnóstico y el tratamiento del síncope. Rev Esp Cardiol. 2018;71(19):837.e1-e92. Disponible en: https://www.revespcardiol.org/es-pdfS0300893218304895? referer=buscador

3. Sheldon RS, Raj S, Predicting Syncope: The First Step in a Long Journey? HRTHM. 2018;15(9):1411-2. doi: 10.1016/j.hrthm.2018.06.002. PubMed Central PIMD PMCID: 29883679

4. Pournazari P, Sahota I, Sheldon R. High Remission Rates in Vasovagal Syncope: Systematic Review and Meta-Analysis of Observational and Randomized Studies. JACC: Clinical
Electrophysiology. 2017;3(4):384-92. Disponible en: https://doi.org/10.1016/j.jacep.2016.10.012

5. Sahota IS, Maxey C, Pournazari P, Sheldon RS. Clusters, Gaps, and Randomness: Vasovagal Syncope Recurrence Patterns. JACC Clin Electrophysiol. 2017;3(9):1046-53. Disponible en: https://doi.org/10.1016/j.jacep.2017.02.008

6. Sheldon RS, Grubb BP, Olshansky B, Shen WK, Calkins H, Brignole M, et al. 2015 Heart Rhythm Society Expert Consensus Statement on the diagnosis and treatment of postural tachycardia syndrome, inappropriate sinus tachycardia, and vasovagal syncope. Heart Rhythm. 2015;12(6):e41-e63. Disponible en: https://doi.org/10.1016/j.hrthm.2015.03.029

7. Rosas F, Jumbo L, Velasco V, Jaramillo C, Rodríguez D. Síncope neurocardiogénico Relación con la edad, género y tipo de respuesta en la prueba de la mesa basculante. Acta Médica Colombiana. 2001;26(2):60-4. Disponible en: http://www.actamedicacolombiana.com/anexo/articulos/0 2-2001-04.htm

8. Baker SE, Limberg JK, Ranadive SM, Joyner MJ. Neurovascular control of blood pressure is influenced by aging, sex, and sex hormones. Am J Physiol Regul Integr Comp Physiol. 2016;311(6):R1271-R1275. Disponible en: https://doi.org/10.1152/ajpregu.00288.2016

9. Limberg JK, Peltonen GL, Johansson RE, Harrell JW, Kellawan JM, Eldridge MW, et al. Greater $\beta$-adrenergic receptor mediated vasodilation in women using oral contraceptives. Front Physiol. 2016;7:215. Disponible en: https://doi.org/10.3389/fphys.2016.00215

10. Briant LJ, Burchell AE, Ratcliffe LE, Charkoudian N, Nightingale AK, Paton JF, et al. Quantifying sympathetic neuro-haemodynamic transduction at rest in humans: insights into sex, ageing and blood pressure control. J Physiol. 2016;594(17):4753-68. Disponible en: https://doi.org/10.1113/JP272167

11. Ng J, Sheldon RS, Ritchie D, Raj V, Raj SR. Reduced quality of life and greater psychological distress in vasovagal syncope patients compared to healthy individuals. Pacing Clin Electrophysiol. 2019;42(2):180-8. Epub 2018 Dec 10. PMID: 30488466; PMCID: PMC6358504. Disponible en: https://doi.org/10.1111/pace.13559

12. Castaño-Morales JA, Lopera-Valle JS, Vanegas-Elorza DA, Cañas-Arenas EM, González-Rivera E. Escala de Calgary para el diagnóstico del síncope vasovagal. Estudio de pruebas diagnósticas. Rev. Colomb. Cardiol. 2015;22(4):162-8. Disponible en: https://doi.org/10.1016/j.rccar.2015.03.007

13. Lee AK, Krahn AD. Evaluation of syncope: focus on diagnosis and treatment of neurally mediated syncope. Expert Rev Cardiovasc Ther. 2016;14(6):725-36. Disponible en: https://doi.org/10.1586/14779072.2016.1164034

14. Piccirillo G, Moscucci F, Fiorucci C, Di Iorio C, Mastropietri F, Magrì D. Time- and frequency-domain analysis of beat to beat P-wave duration, PR interval and RR interval can predict asystole as form of syncope during head-up tilt. Physiological Measurement. 2016;37(11):1910-24. Disponible en: https://doi.org/10.1088/09673334/37/11/1910

15. Keller K, Beule J, Balzer JO, Dippold W. Syncope and collapse in acute pulmonary embolism. Am J Emerg. Med. 2016;34(7):1251-7. Disponible en: https://doi.org/10.1016/j.ajem.2016.03.061

16. Sheldon R. Syncope Diagnostic Scores. Prog Cardiovasc Dis. 2013;55(4):390-5. Disponible https://doi.org/10.1016/j.pcad.2012.10.011 
17. Kim TH, Jang HJ, Kim S, Cho SY, Song KS, Pickett C, et al. A new test for diagnosing vasovagal syncope: Standing after treadmill test with sublingual nitrate administration. PLOS One. 2017;12(6):e0179631. Disponible en: https://doi.org/10.1371/journal.pone.0179631

18. Asociación Médica Mundial. Declaración de Helsinki de la Asociación Médica Mundial (AMM) principios éticos para las investigaciones médicas en seres humanos. 64a Asamblea General. 2013. Disponible en: https://www.wma.net/es/policies-post/declaracion-dehelsinki-de-la-amm-principios-eticos-para-lasinvestigaciones-medicas-en-seres-humanos/. Consultado 03 Mar 2018.

19. Chaturvedi A, Kumar A. Syncope: Clinical study and outcome of diagnostic evaluation. J Assoc Physicians India. 2018 Jan;66(1):37-40.

20. Sheldon R, Rose MS, Ritchie D, Martens K, Maxey C, Jagers J, et al. Genetic Association Study in Multigenerational Kindreds With Vasovagal Syncope. Circ Arrhythm Electrophysiol. 2019;12(1):e006884. PubMed PMID:30636478. Disponible en: https://doi.org/10.1161/CIRCEP.118.006884

21. Skoog J, Zachrisson H, Länne T, Lindenberger M. Reduced compensatory responses to maintain central blood volume during hypovolemic stress in women with vasovagal syncope. Am J Physiol Regul Integr Comp Physiol. 2017;312(1):R55-R61. Disponible en: https://doi.org/10.1152/ajpregu.00166.2016

22. Christou GA, Kouidi EJ, Anifanti MA, Sotiriou PG, Koutlianos NA, Deligiannis AP. Pathophysiological mechanisms of noncardiac syncope in athletes. Int J Cardiol. 2016;224:20-6. Disponible https://doi.org/10.1016/j.ijcard.2016.08.308

23. Abizanda P, Garcia I, Luengo C, et al. Tratado de Geriatría para Residentes, 1aㅡ Edición. En: Pedro Abizanda Soler, M.a Isabel García Tomás, Editores, Situaciones clínicas Relevantes, Capítulo 32: Síncope, Sociedad Española de Geriatría y Gerontología (SEGG), España: Editorial: International Marketing \& Communication, S.A. (IM\&C), 2017. p. 333-42.

24. Stewart JM, Medow MS, Sutton R, Visintainer P, Jardine DL, Wieling W. Mechanisms of Vasovagal Syncope in the Young: Reduced Systemic Vascular Resistance Versus Reduced Cardiac Output. J Am Heart Assoc. 2017;6(1):e004417. Disponible en: https://doi.org/10.1161/jaha.116.004417

25. Flevari P, Leftheriotis D, Kroupis C, Antonakos G, Lekakis J, Dima K. Copeptin levels in patients with vasovagal syncope. Int. J. Cardiol. 2017;230:642-5. Disponible en: https://doi.org/10.1016/j.ijcard.2017.01.014

26. Marechal P, Scheen AJ, Lancellotti P. Syncope aux Urgences Diagnostic différentiel et stratification du risque. Rev Med Liege. 2018;73(5-6):237-42. Disponible en: http://hdl.handle.net/2268/230421

27. Angus S. The Cost-Effective Evaluation of Syncope. Med Clin N Am. 2016;100(5):1019-32. Disponible from: https://doi.org/10.1016/j.mcna.2016.04.010

28. Palaniswamy C, Aronow WS, Agrawal N, Balasubramaniyam N, Lakshmanadoss U. Syncope: Approaches to Diagnosis and Management. Am J Ther. 2016;23(1):208-17. Disponible en: https://doi.org/10.1097/MJT.0b013e318256ed0f 